\title{
La tradición camaleónica en las prácticas festivas de una comunidad mixteca transnacional
}

\section{Chameleonic Traditions in the Festive Practices of a Mixtec Transnational Community}

\begin{abstract}
Resumen
¿En qué consiste la práctica de la "tradición” entre las comunidades indígenas? Este artículo está enfocado en un análisis etnográfico de las prácticas festivas de la comunidad mixteca transnacional de San Juan Mixtepec, Oaxaca, en México, a la luz del concepto antropológico de "tradición". El artículo investiga las relaciones presentes entre la creación, el mantenimiento y la transformación en la práctica de las tradiciones, esto a través de un análisis que toma en cuenta algunas de las prácticas festivas de esta comunidad mixteca. Específicamente, el artículo plantea la tradición como un concepto "camaleónico" que reside en el espacio entre la permanencia y la transformación, dando una base histórica o conmemorativa a las experiencias y recuerdos de los participantes, al tiempo que sirve como contexto dinámico para la exploración de novedosas construcciones de identidades. Es así que, el artículo propone un nuevo modelo analítico para la investigación de las comunidades indígenas transnacionales, donde las tradiciones están vistas como un catalizador para la construcción de sentidos de pertenencia en un contexto etnográfico donde las experiencias cotidianas de las personas están marcadas, en muchos sentidos, por el movimiento.

Palabras claves: Comunidades transnacionales, Tradición y performatividad, Cambio cultural, Pertenencia comunitaria, Cultura mixteca
\end{abstract}

\begin{abstract}
What does it mean to practice "tradition" in indigenous communities? This article focuses on an ethnographic analysis of celebrations in the Mixtec transnational community of San Juan Mixtepec, Oaxaca, Mexico in the light of the anthropological concept of "tradition". This article investigates the relationship between the creation, maintenance, and transformation of the practice of tradition by the analysis of some of the celebrations of this Mixtec community. Specifically, the article presents tradition as a "chameleonic" concept which resides in the space between permanence and transformation, providing an historic or commemorative foundation for the practices and memories of its participants and working as a dynamic context for the exploration of new identity constructions. In this way, this
\end{abstract}

1. Universidad Autónoma de San Luis Potosí, México, Profesora-Investigadora, Coordinadora de la Licenciatura en Antropología en la Facultad de Ciencias Sociales y Humanidades, ivy. rieger@uaslp.mx

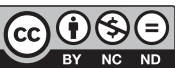


article proposes a new analytic model for the investigation of indigenous transnational communities, where traditions are portrayed as a catalyst for senses of belonging in an ethnographic context where daily experiences are marked, in many ways, by movement.

Key Words: Transnational communities, Tradition and performativity, Cultural change, Community belonging, Mixtec culture

\section{Introducción}

“Tradición” es un concepto que tiene una larga trayectoria en los estudios antropológicos (BenAmos, 1984; Hobsbawm y Ranger, 1983; Noyes, 2009; Toren, 1988). Es un concepto que ha englobado un sinfín de prácticas relacionadas con la producción y con el mantenimiento de discursos culturales, los cuales incluyen una dimensión histórica que habla de su supuesta antigüedad. En pocas palabras, para que una práctica se pueda clasificar como una tradición, hay que contar primero con una conceptualización de la historia, sea escrita u oral. Esta "historia" puede ser documentada o imaginaria, pero en ambos casos funge como un elemento unificador para los participantes, dándoles, en muchos casos, una base para la construcción de identidades.

La tradición también tiene una característica de "performatividad" en el sentido de que su enseñanza continua depende muchas veces de la realización de prácticas corporizadas, las cuales transmiten conocimiento a través de la imagen corporizada y de la expresión oral. Muchas tradiciones son practicadas en ámbitos sociales, donde el mismo cuerpo humano sirve como un lienzo para la transmisión de las ideologías socioculturales. La manera en que se define la ‘tradición' nace entonces, principalmente, de estos dos ejes (histórico y performativo) y está vinculada con un sentido de proceso o de progresión, el cual estructura el camino para la transmisión de las prácticas y de los conocimientos materiales e inmateriales, que define lo que cuenta (y lo que no) como "tradicional".

No obstante, cabe preguntar más allá de las explicaciones académicas del concepto de "tradición”: ¿qué utilidad tiene este término para los propios practicantes de estas tradiciones? El artículo está enfocado en una exploración de las relaciones presentes entre el concepto antropológico de "tradición" y la conceptualización indígena del término a través de un análisis de algunas de las prácticas festivas de la comunidad mixteca de San Juan Mixtepec, Oaxaca, en México. En San Juan Mixtepec, las fiestas están integradas en, aparentemente, todos los aspectos de la vida social. Sus miembros, quienes viven dentro y fuera de la comunidad, salvaguardan su ciclo de fiestas, las cuales católicos particulares celebran a santos y a vírgenes, así como una serie de fiestas que realizan rituales, representaciones, y actividades específicas asociadas con creencias cosmológicas mixtecas. La mayoría de las comunidades mixtecas de Oaxaca, aunque celebran su fiesta patronal y otras fiestas importantes como la Semana Santa, no tienen un ciclo completo de fiestas debido a niveles cada vez mayores de emigración y otros cambios socioeconómicos (Alcalá y Couturier, 1994; Cornelius et al., 2011; Hernández-Díaz, 2011). A diferencia de otros estudios, los cuales se han enfocado en diferentes aspectos del fenómeno de migración, este artículo aporta un modelo analítico sobre la pertenencia en comunidades mixtecas transnacionales. Específicamente, se propone que el caso de San Juan Mixtepec resulta único entre muchas comunidades mixtecas porque, a pesar de altas cifras de migración externa, sus fiestas siguen creando un loccus vital de orientación social y de producción de identidad cultural, además de representar un elemento crítico para la conceptualización de sentidos de pertenencia para sus habitantes.

La elección de San Juan Mixtepec como la comunidad de trabajo obedece precisamente a la complejidad de su ciclo festivo presente a pesar de, o debido a, la influencia constante del fenómeno de

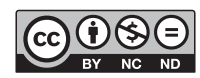


migración. El artículo se basa en materiales etnográficos realizados entre los años 2013 y 2017 en dicha comunidad y una de sus comunidades de diáspora ubicada en Bakersfield, California, Estados Unidos. Los primeros encuentros ocurrieron en 2009 y 2011, cuando llevé a cabo proyectos predoctorales para familiarizarse con las prácticas festivas de la comunidad.

La investigación doctoral consistía en una estancia de campo de un año (2013) y estancias cortas de aproximadamente un mes cada una (durante el 2016 en Oaxaca y durante el 2017 en California) enfocadas en la documentación fotográfica, videográfica y en análisis etnográfico del ciclo completo de las fiestas religiosas. Muchos conceptos y términos sobre las tradiciones festivas no cuentan con una traducción al español, por lo cual aprendí un nivel básico de la variante mixtepequense del mixteco como parte de mi investigación ${ }^{1}$. La investigación de las fiestas se completó con entrevistas de las personas migrantes y residentes de la comunidad, enfocadas en temas relacionados con las tradiciones presentes en las fiestas, sus recuerdos de fiestas pasadas y la organización y relaciones políticas presentes en las instituciones involucradas; tales como, historias de vida para documentar los cambios en la práctica de las fiestas, así como los cambios generales experimentados en la comunidad.

La investigación del ciclo de las fiestas revela que esta tradición ha permanecido como una de las principales prácticas sociales para los residentes y migrantes de San Juan Mixtepec. Específicamente, estas festividades dan forma a las maneras en que los miembros de la comunidad, referidos de aquí en adelante como "los mixtepequenses" ${ }^{2}$, practican lo que significa ser parte de esta comunidad indígena más allá de las fronteras. Es así que, el artículo analiza el concepto de "tradición" precisamente como un elemento clave en la producción de los sentidos de pertenencia para los miembros y migrantes de esta comunidad, por lo tanto, no solamente se trata de cuestiones relacionadas con la preservación de costumbres culturales, sino también con el concepto del cambio y con la transformación de identidades. Así, una de las tesis principales del artículo es que las fiestas son prácticas camaleónicas, es decir prácticas dinámicas que se caracterizan tanto por sus elementos permanentes como por sus elementos novedosos (estos cambian dependiendo en las experiencias de las participantes). Es decir, la fiesta en sí cuenta con una estructura u organización relativamente fija, pero las prácticas presentes y las formas que toman son cambiantes. Basada en esta idea, la investigación de las fiestas de San Juan Mixtepec se enfoca en la naturaleza multifacética de la práctica de tradiciones para ofrecer un modelo analítico relativo a la práctica de pertenencia en ésta y otras comunidades indígenas transnacionales. 


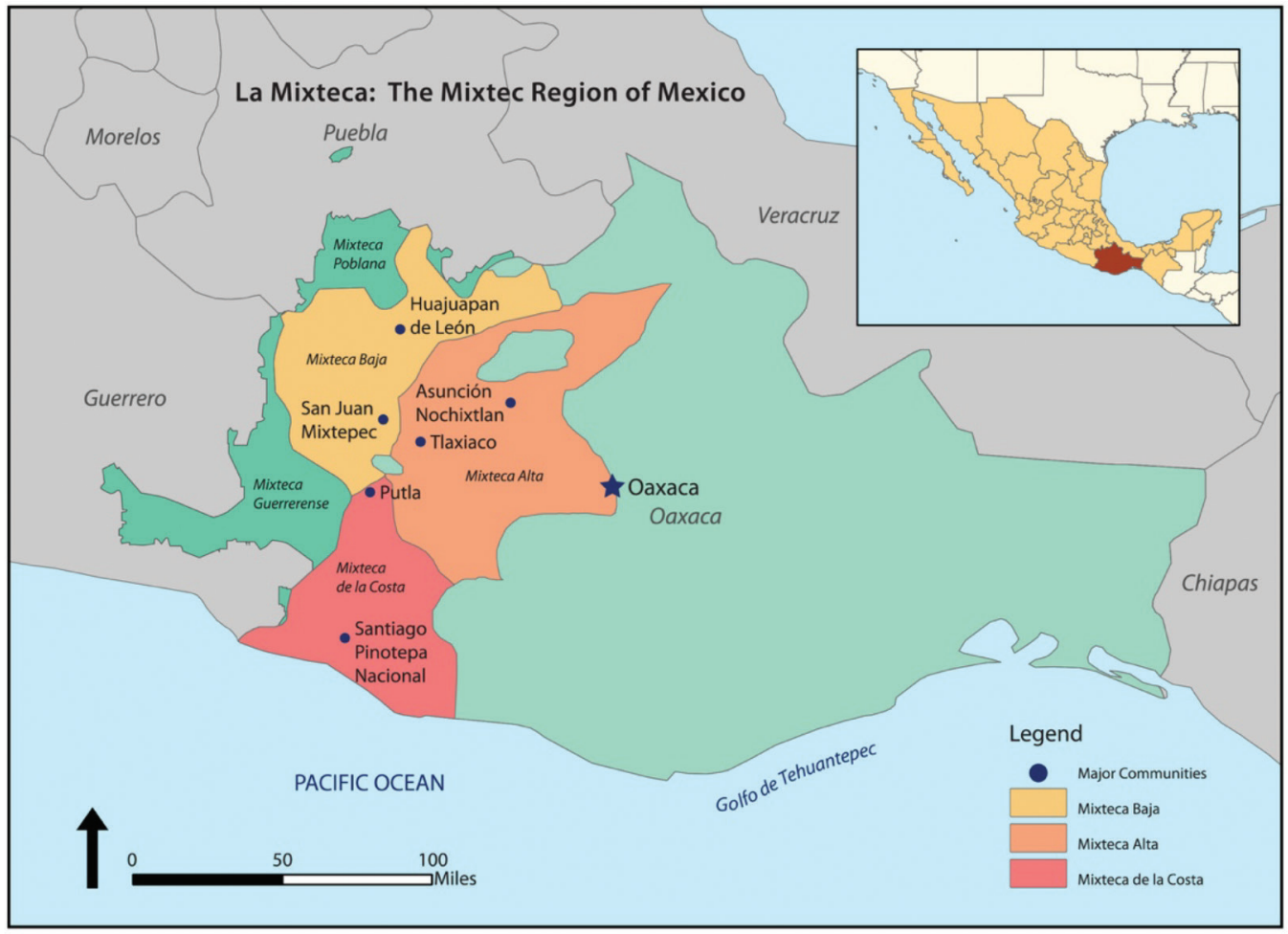

Fuente: Mapa elaborado por la persona autora.

\section{Los mixtecos de $\tilde{N} u u$ Snuviko (San Juan Mixtepec)}

Los mixtepequenses pertenecen al grupo indígena mexicana conocido como los mixtecos, o los nivi ñuu savi ("gente del pueblo de la lluvia"). Ellos se identifican a través de una red cultural compartida colectivamente basada en características lingüísticas, geográficas, sociales, arqueológicas e históricas relacionadas con los valores, el lenguaje, la historia y la organización social de una sociedad prehispánica también conocida como "los mixtecos". La región geográfica original de los mixtecos está ubicada en la parte sur del estado de Puebla, la región oriental del estado de Guerrero y hacia la mitad occidental del estado de Oaxaca, donde la mayoría de los mixtecos residen hoy en día.

Dentro del estado de Oaxaca, la región mixteca está dividida en tres áreas geográficas distintas: la Mixteca Alta, la Mixteca Baja y la Mixteca de la Costa (Figura 1). Durante el periodo clásico (A.D. 200-900) y posclásico (A.D. 900-1520), el desarrollo de lo que los arqueólogos e historiadores clasifican como "la cultura mixteca" ocurrió principalmente en la intersección de estas tres regiones (Joyce 2010; Terraciano 2001). San Juan Mixtepec está ubicado en la frontera entre las regiones de la Mixteca Baja y la Mixteca Alta y alude tanto al municipio de San Juan Mixtepec (el cual incluye 61 pueblos, rancherías y otras dependencias) así como a la cabecera municipal del mismo nombre. De acuerdo con el censo

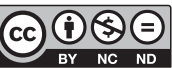


2010, la población de San Juan Mixtepec contaba con 7,611 habitantes, de los cuales más del 93\% de la población municipal eran hablantes de la variante mixtepequense del idioma mixteco ${ }^{3}$.

La cabecera municipal está compuesta de seis distintos barrios: San Miguel Centro, San Miguel Lado, San Pedro, San Sebastián, Barrio de Jesús y Barrio Centro. Una de las principales maneras mediante las cuales los mixtepequenses conceptualizan sus sentidos de pertenencia es a través de la afiliación con un barrio en particular como su lugar de nacimiento o de residencia actual, lo cual está típicamente asociado con la presencia histórica de su linaje familiar en el barrio. Los barrios de San Sebastián, San Pedro y San Miguel Lado también cuentan con su propia capilla donde los residentes realizan servicios religiosos asociados con las imágenes correspondientes, allende su participación en las actividades de la iglesia municipal de San Juan Bautista, ubicada en el Barrio Centro. La afiliación con un barrio es central en la manera en que los mixtepequenses participan en las diferentes instituciones sociales y políticas, mismas que forman parte de su vida cotidiana y religiosa, como el tequio ${ }^{4}$, la mayordomía ${ }^{5}$ o los comités comunitarios ${ }^{6}$.

San Juan Mixtepec es una comunidad situada en un valle aluvial fértil donde se cruzan el Río Mixteco y el Río Chiquito, dentro de las montañas de la Sierra Madre del Sur. Los residentes del municipio siguen practicando técnicas agrícolas tradicionales en sus cultivos de maíz, frijol y calabaza en los jardines del traspatio, así como en grandes milpas de varias hectáreas. Crían ganado (toros, vacas, borregos, chivos, marranos, gallinas y guajolotes) como parte del mercado local, así como para el consumo doméstico. Además de ser propietarios de terrenos agrícolas, muchos individuos y familias son dueños de una variedad de negocios locales. Algunos mixtepequenses se trasladan a otras ciudades para trabajar durante la semana como profesionistas u obreros en una variedad de oficios en lugares como Tlaxiaco, Juxtlahuaca o la cuidad de Oaxaca. Sin embargo, la mayoría participa en procesos de migración regional, nacional, y transnacional, procesos que cuentan con una larga historia en esta comunidad indígena (Besserer y Kearney, 2006; Edinger, 2004; Ruvalcaba y Robles, 2012).

Por lo menos desde la década de los 30's los mixtepequenses establecieron sus primeras comunidades modernas de diáspora en lugares como Abasolo del Valle, Veracruz, la Cuidad de México y en Bakersfield, California, así como en los estados mexicanos de Sinaloa y Baja California y en los estados norteamericanos de Florida, Carolina del Norte, Washington, Oregón y Arizona, entre otros. La migración es un proceso y una práctica que inciden en la naturaleza de conceptos tales como "identidad" y "pertenencia" en ésta y otras comunidades indígenas (Cornelius et al., 2017; Nagengast y Kearney, 1990; Lestage, 2011; Ravicz, 1965; Velasco Ortíz, 2005). Los mixtepequenses residentes en los Estados Unidos administran múltiples identidades culturales, sociales y políticas y una gran mayoría son trilingües (mixteco, español e inglés).

Las fiestas de San Juan Mixtepec forman una parte intrínseca de este panorama social. Los mixtepequenses practican un ciclo completo de trece fiestas religiosas ${ }^{7}$ y tres fiestas seculares ${ }^{8}$ durante el año, las cuales celebran santos y vírgenes de forma específica, así como algunas fechas importantes en el calendario cosmológico mixteco. Las fiestas de San Juan Mixtepec están dirigidas y organizadas, principalmente, por parte de la mayordomía y en ciertos casos - como en el caso de la fiesta patronal de San Juan Bautista y el Kastalenche (Carnaval)- en coordinación con las autoridades municipales.

La institución de la mayordomía forma parte del lado religioso de la jerarquía civil-religiosa de la comunidad cuyo objetivo práctico es, en términos generales, organizar la celebración de los santos y de las vírgenes católicas presentes en la iglesia. Este cargo está compuesto de diferentes elementos, los cuales los mayordomos están obligados a cumplir durante su mandato de un año.

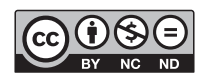


Entre las responsabilidades de los mayordomos se encuentran: 1) el mantenimiento y cuidado de las imágenes físicas, los nichos y los altares de los santos, así como el aseo general de la iglesia y atrio; 2) la organización de las fiestas de su santo y sus rituales asociados; 3) formar parte de un consejo que está dedicado a tratar los asuntos religiosos de la comunidad; 4) la administración de los recursos económicos y ahorros asociados con su santo (también conocido como "el cargo" del santo); 5) participar en las fiestas de las demás mayordomías; $y, 6$ ) fungir como representantes de su santo en otras prácticas religiosas de la parroquia, como las misas, los bautismos y las bodas, entre otras tareas.

Cada fiesta religiosa consiste en la celebración de una imagen en particular, con una duración que va de entre dos y tres días, con un horario específico de actividades religiosas. Dichas actividades combinan las siguientes características: vísperas, misas, procesiones, comidas en la casa del mayordomo, bailes y rituales mixtecos, entre otras. Las fiestas seculares también duran entre dos y tres días y pueden incluir la mayoría de estas características, con excepción de los elementos religiosos católicos. Estas fiestas están entrelazadas con muchos otros elementos de la vida social, como la organización socio-política, los discursos culturales, las prácticas religiosas y la preservación y práctica del patrimonio cultural como: la gastronomía, la música de viento y de cuerdas, las danzas, los disfrazados y los rituales indígenas. También existe la práctica de fiestas caseras que tienen que ver con tradiciones relacionadas con ritos funerarios, las bodas, los bautismos y fiestas de primera comunión, entre otras.

Las fiestas principales del ciclo dependen, en mayor parte, de la participación activa de las personas migrantes, quienes regresan a San Juan Mixtepec para fungir como mayordomos, participantes, voluntarios, y miembros del público. La población migrante también proporciona un apoyo económico sustancial al proyecto de la fiesta a través de las donaciones directas a las mayordomías o a los comités y a la comunidad en sí, a través de puestos de comida y de tiendas locales durante la fiesta. Además, los migrantes toman fotos y videos que luego suben a las redes sociales para promover, activa o inactivamente, la fiesta como una parte importante de las construcciones contemporáneas de identidades y de los sentidos de pertenencia. En las fiestas también pueden influir las prácticas de los migrantes, quienes hacen viajes periódicos a Oaxaca, específicamente, para participar en la fiesta y también han migrado su fiesta patronal a sus lugares de destino en tierras lejanas. Las prácticas de la fiesta entre los migrantes, así como entre los residentes permanentes de esta comunidad demuestran que dicha práctica parece estar vinculada directamente con un concepto de tradición, los sentidos de pertenencia y la construcción de identidades.

\section{Historia y tradición en la práctica de las fiestas mixtecas}

¿Dónde nace una tradición y cómo podemos, como académicos y como practicantes de la misma, identificarla? Un elemento principal en la teorización sobre las tradiciones insiste en que el concepto de "historia" es la base para la creación de tradiciones. En pocas palabras, sin una referencia al pasado, las tradiciones no pueden nacer. Esta característica histórica está ligada con la experiencia, donde "la tradición, sujeta a ciertas modificaciones, tiene su origen en la relación dialéctica entre el pasado y el presente, entre la continuidad y el cambio" (Arévalo, 2004, p. 927). Esta relación dialéctica depende de las experiencias colectivas e individuales de los actores sociales que forman parte del desarrollo del concepto de "tradición". De esta manera, la tradición no puede ser concebida como algo estático, sino como algo ligado a un concepto de "cambio", basado en las perspectivas y en las experiencias de las personas participantes acerca de sus propias percepciones sobre el pasado. Es así como, las tradiciones aparecen, se transforman y desaparecen de acuerdo a las relaciones que los mismos practicantes tengan con el concepto de "historia" (Anttonen, 2016; Ó Giolláin, 2000). Como explica Henry Glassie (1995,

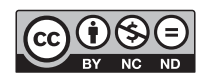


p. 396) "si la tradición es una creación de la gente de su propio pasado, su carácter no es de estasis, sino de continuidad; su opuesto no es el cambio sino la oposición, la intrusión de un poder que frustra el curso del desarrollo"".

Para construir un concepto de tradición primero es fundamental investigar las maneras a través de las cuales los mismos participantes conciben sus relaciones con el pasado. En mis investigaciones (Rieger, 2015, 2017, 2018) con los mixtepequenses, las relaciones presentes entre la historia y la tradición parecen concentrarse, principalmente, en dos ejes que interactúan entre sí: la transmisión oral y la participación activa.

El primer eje, la transmisión oral, se refiere a los intercambios verbales de los conocimientos culturales. Son principalmente los mayordomos anteriores y los ancianos de la comunidad quienes comparten sus conocimientos a través del intercambio de sus experiencias en el contexto festivo, lo cual, generalmente, está orientado en sus recuerdos sobre cómo se practicaba la fiesta en años anteriores. Estas personas típicamente guían a los participantes respecto a los pasos específicos que tienen los rituales, las procesiones, la matanza de los animales, entre otras actividades. La información puede ser compartida en español, pero es más común observar el uso del mixteco en estos contextos específicos. Cuando estas personas mueran, si no tienen un aprendiz, dicho conocimiento se perderá. Sin embargo, es importante recordar que la transmisión oral de las tradiciones no está exclusivamente limitada a estas personas, sino que también incluye a los migrantes que llevan estos conocimientos a sus comunidades de diáspora.

Como señala Graburn (2008, p. 6) en su resumen de la obra de Horner (1990): "la tradición se refiere tanto al proceso de transmisión de generación en generación y a alguna cosa, costumbre o proceso de pensamiento que es transmitido en el tiempo" ${ }^{10}$. Los mixtepequenses se involucran en una red de transmisiones conscientes e inconscientes de sus prácticas socioculturales donde están expuestos, o activamente participan, en la generación, la transmisión y la preservación de conocimientos sobre sus tradiciones. El sistema de cargos $^{11}$ en la comunidad facilita el mantenimiento de esta memoria colectiva, la cual sirve como una "base de datos" no escrita sobre las tradiciones e historia de las prácticas religiosas de la comunidad.

Los mayordomos, quienes pueden ser considerados como los guardianes de la memoria colectiva, resguardan mucha de la historia de las tradiciones festivas a través de su práctica, y no a través de escrituras o archivos, más allá de los libros mayores de los gastos económicos de cada mayordomía. Este detalle es importante porque destaca la naturaleza dinámica de la transmisión oral y de la práctica de tradiciones como las fiestas y enfatiza la manera en la que su preservación depende, principalmente, de la continuidad de ciertas prácticas performativas (como en el caso de la fiesta de San Juan Bautista, analizado en la siguiente sección). Estas prácticas transmiten discursos específicos acerca de lo que las personas participantes, las organizaciones sociales y la comunidad en sí, consideran digno de recordar. El concepto de tradición depende de la transmisión efectiva de estos discursos porque, en parte, "la calidad de ser tradicional es una percepción entre participantes que su acción es el resultado de conexiones sociales las cuales han perdurado a través del espacio y el tiempo" (Howard, 2013, p. 73) ${ }^{12}$. En efecto, muchos mixtepequenses aprenden sobre las tradiciones de su comunidad a través de la digestión activa o pasiva de estos discursos presentados por parte de los demás participantes en las tradiciones festivas.

El vínculo entre las fiestas y el concepto de historia en San Juan Mixtepec depende principalmente de procesos de transmisión, selección y transformación, como señalaba Arévalo: "la tradición no se hereda genéticamente; se transmite socialmente y deriva de un proceso de selección cultural" (2004, p. 927). La mayordomía es uno de los medios principales de transmisión de la historia de las tradiciones en San Juan Mixtepec. La gente "sabe" cómo hacer una fiesta precisamente basada en sus propios

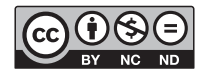


recuerdos de fiestas anteriores y con base en la memoria colectiva compartida dentro de la mayordomía al momento de organizar la fiesta. Sin embargo, su éxito también depende de un conocimiento compartido con los demás miembros de la comunidad, quienes son externos a la mayordomía, incluyendo a los ancianos, los chaa ka'a tsavi ${ }^{13}$, migrantes o exmayordomos, entre otros. Por ende, cada fiesta cuenta con expresiones distintas derivadas de los recuerdos y preferencias de cada mayordomo, aunque la estructura general de la fiesta es la misma. Es este sentido de reciprocidad en la transmisión de conocimientos sobre las características de las fiestas lo que alimenta su práctica, además de los sentidos de pertenencia inculcados dentro de su esfera.

La historia, así como el concepto de tradición, es algo dinámico en la práctica de las fiestas de San Juan Mixtepec. Como se mencionó anteriormente, las fiestas no cuentan con una historia escrita de su práctica, ni con una guía que destaque cuáles son las características que deben aparecer en cada una. La transmisión de conocimientos depende, principalmente, de una enseñanza práctica o de indicaciones orales: recuerdos individuales y colectivos, interpretaciones individuales de los participantes, etc. En mis entrevistas con participantes de las fiestas, varios señalaron algo que podemos definir como una concepción propia de la historia, la cual está ligada más con una memoria colectiva que con hechos o eventos precisos del pasado (Rieger, 2018). Además, muchos mixtepequenses notaron que una de las razones principales por las que siguen practicando las fiestas es que las consideran como "la tradición de mi pueblo". La práctica de la fiesta en el presente parece ser lo que cuenta como historia y como tradición para muchos miembros de esta comunidad indígena.

Por lo tanto, hay algunas prácticas que responden a un apego a lo que los participantes consideran parte de su "tradición" a pesar de, o incluso debido a, la gran influencia de la migración. Aunque vivan en el extranjero, por ejemplo, cada adulto está obligado a cumplir con sus responsabilidades sociales, tales como participar en la mayordomía o en los otros cargos comunitarios. De lo contrario, los miembros de su barrio pueden aplicar ciertos castigos sociales, como suspender los servicios de electricidad y de agua a sus propiedades, o amenazar con que uno de sus familiares quedará comprometido a tomar el cargo bajo su nombre si no se responsabiliza. No es extraño escuchar casos de migrantes que interrumpen sus vidas en los Estados Unidos, por lo menos un año o más, para regresar a Oaxaca y participar como miembros activos de la comunidad en estos cargos, sin importar su situación migratoria. Esto habla de la importancia del concepto de continuidad en la práctica de tradición y en la transmisión activa de los conocimientos culturales por parte de los actores involucrados en los cargos de la comunidad.

El segundo eje es la participación activa o performativa, la cual complementa la transmisión oral y se refiere a las prácticas corporizadas que se llevan a cabo principalmente durante las fiestas. Una de las maneras en que el conocimiento sobre la fiesta, y sobre las tradiciones en general, se comparte, preserva y transforma, es a través del uso del propio cuerpo como un lente performativo que transmite los discursos culturales. El uso del cuerpo es evidente en la práctica de las tradiciones por parte de todos los participantes en las fiestas de San Juan Mixtepec, en tanto participantes de los diferentes rituales, procesiones y otras actividades asociadas con el contexto festivo (como los disfrazados, analizados abajo).

De acuerdo con Herrejón Peredo "la acción de entregar, de transmitir, implica un agente. El agente de la tradición es el hombre...y como el agente de la tradición no es únicamente el hombre como individuo y persona, sino el hombre sociedad y comunidad...se trata, pues, de un grado de conciencia y querer colectivos" (1994, p. 137). El agente es por lo menos un cuerpo físico, un participante o un actor social en algunos casos, que utiliza ciertas prácticas específicas, las cuales tienen la capacidad de transmitir tradiciones a través de discursos simbólicos corporales. En el contexto de la fiesta, este proceso de transmisión corporal es lo que da vida a la práctica de tradiciones para muchos residentes y migrantes de San Juan Mixtepec.

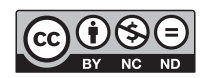


Además, estos procesos están sujetos a transformaciones debido a las interpretaciones específicas por parte de quienes los practican. Por ende, la tradición debe ser concebida como "un proceso de transmisión activo, es decir, la tradición viva, la que se ubica en el tiempo actual, fruto de una serie de reiteraciones del contenido entregado, el cual no se conserva idéntico, sino que es susceptible a ser modificado parcialmente, y está abierto a los impulsos creativos de sus detentadores" (Madrazo Miranda 2005, p. 123). Esta transmisión se lleva a cabo, principalmente, a través de acciones corporales de los participantes e interpretados por parte de los demás, quienes los observan en el contexto festivo en el presente.

Por ende, podemos inferir que los elementos presentes en el ciclo tradicional de las fiestas forman parte de una práctica de "tradicionalización", la cual está definida como "un proceso que evoca el pasado tradicional, no como parte de un diálogo general con el pasado, sino como un intento de otorgar autoridad al performance narrativo y a la interpretación del individuo a través de apoyar o contribuir al sentido comunitario de lo que define lo tradicional" (Mould 2005, p. 257) ${ }^{14}$. Específicamente, es a través de la interpretación corporal que los participantes en las fiestas dan vida a la tradición en sí, y es en estos contextos que las posibles transformaciones o cambios a las tradiciones se llevan a cabo. El uso del propio cuerpo, que sea humano o animal, es clave en la transmisión visual de las tradiciones, siendo un elemento importante en la enseñanza de este concepto al público. Además, el uso del cuerpo como vehículo de las tradiciones proporciona una plataforma individualizada donde las personas participantes pueden "jugar" con las características específicas de las prácticas a través de la personalización.

\section{La tradición camaleónica en la práctica de las fiestas mixtecas}

Es principalmente a través de las relaciones presentes entre estos dos ejes de transmisión que podemos observar cómo las fiestas reflejan los procesos de cambio y de continuidad en la práctica de las tradiciones, estos procesos están íntimamente ligados con las experiencias de vida de los residentes y migrantes de la comunidad. Esto es lo que hace de la tradición algo vivo, algo que sirve como una base para la orientación social, como un catalizador para posibles transformaciones a nivel individual y colectivo. Si la tradición "se refiere a un proceso interpretativo, que encarna la continuidad y la discontinuidad" 15 , es imposible pensar que una tradición, aunque cuenta con ciertas características específicas y una historia o memoria colectiva de su práctica, es estática, especialmente cuando la práctica de dichas tradiciones ocurre en un contexto social migratorio (Handler y Linnekin 1984, p. 273). Más bien, hay que pensar en cómo identificar las maneras a través de las cuales los mismos miembros de las comunidades indígenas interactúan con los conceptos antropológicos de historia, de identidad y de autenticidad en la práctica de sus tradiciones. Dada la práctica de sus fiestas, es posible afirmar que una dialéctica entre estos conceptos está presente, particularmente en lo relativo a tres temas en particular: la preservación cultural, la identidad mixteca contemporánea y la migración transnacional.

A lo largo de los años que he trabajado en la comunidad, he podido observar las dinámicas de transformación y de continuidad en el ciclo festivo de San Juan Mixtepec, las cuales dan testimonio de la naturaleza camaleónica de las prácticas festivas. Por ejemplo, los elementos presentes en las fiestas, particularmente, en la fiesta patronal de San Juan Bautista, cambia prácticamente cada año de acuerdo a los gustos del mayordomo tal como los de las autoridades municipales, pero también tienden a mantener la misma estructura general.

La fiesta de San Juan Bautista es un asunto de tres días, que empieza con las vísperas el día 23 de junio, seguido por la misa principal, el primer ritual de la despescuezada de gallos ${ }^{16}$ (Figura 2), los bailes de los disfrazados y las comidas colectivas en la casa del mayordomo el día 24 . Terminando con otra misa, otro ritual de gallos, y otros bailes y comidas el día 25 de junio. Los participantes siempre

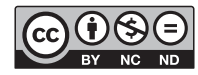


realizan los mismos rituales, procesiones y actividades, siguiendo el mismo orden, porque estas actividades forman parte de las creencias cosmológicas de la comunidad acerca de la adoración del santo, tal como la celebración de los dioses mixtecos de la lluvia.

Sin embargo, una novedad que ha aparecido desde el año 2013 y que se desvía de esta fórmula, es la inclusión de un programa cultural, o calenda, con elementos parecidos a los de la Guelaguetza, celebrada en la ciudad de Oaxaca. Dicho programa ha incluido presentaciones de danza folklórica que ejecutan danzas típicas de las ocho regiones del estado durante las actividades municipales, así como el uso de los "monos de calenda" -muñecos gigantes en formas evocadoras de seres humanos, hechos de papel maché- durante los bailes y procesiones asociados con la mayordomía.

Figura 2

Jinetes y público durante la despescuezada de gallos

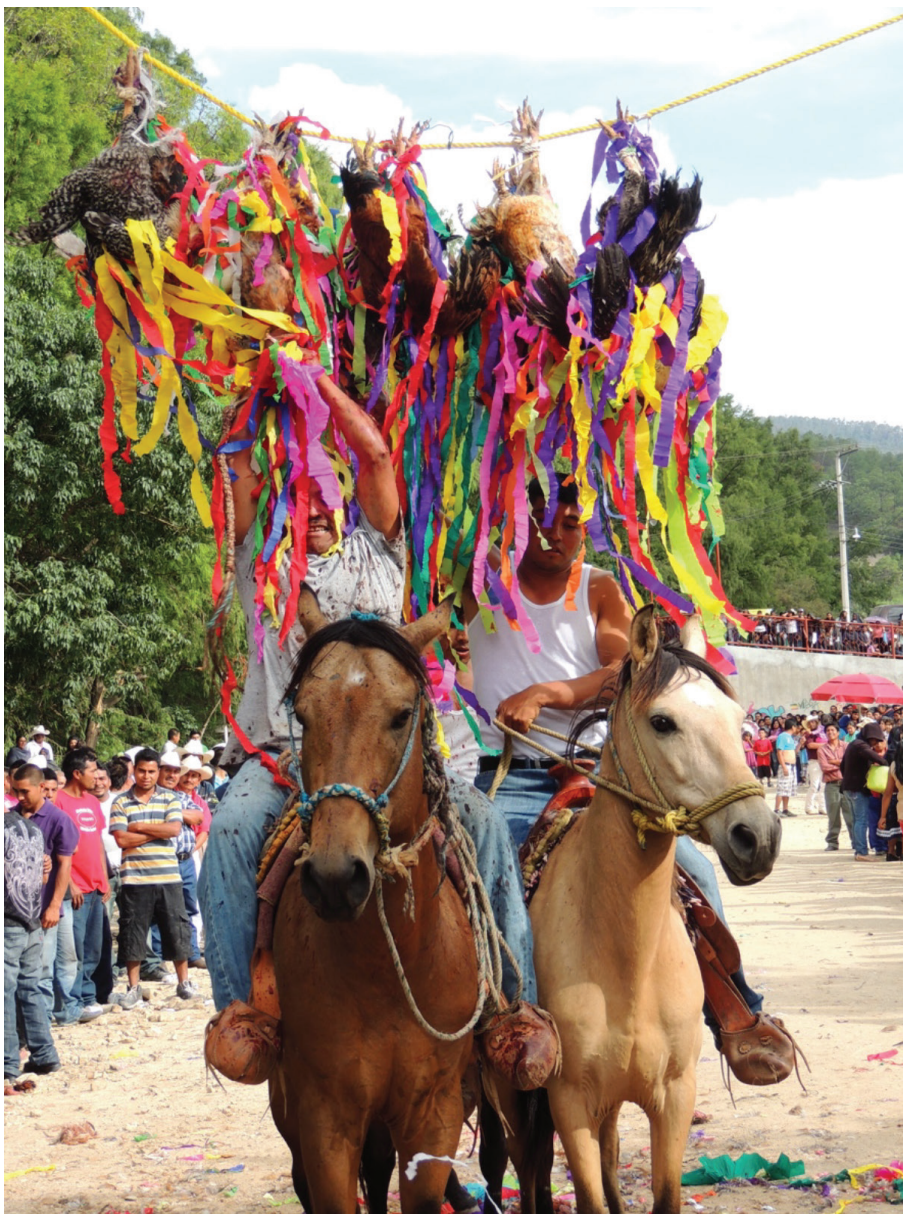

Fuente: Fotografía tomada por la persona autora en San Juan Mixtepec, junio de 2013.

Cabe destacar que, cuando pregunté a la mayordoma de San Juan Bautista, quien es migrante, por qué decidió usar elementos asociados con la Guelaguetza, me comentó que "es importante incluir cosas que

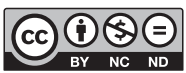


son parte de nuestra cultura oaxaqueña". Es interesante que la misma mayordoma también insistiera en que los mayordomos dejaban de usar el traje de "ranchera" durante su fiesta, el cual consiste en faldas, pantalones, camisas y sombreros de vaquero hechos de materiales sintéticos, en favor de los trajes "tradicionales" de San Juan Mixtepec hechos de algodón y lana. Además, durante las más recientes fiestas de San Juan Bautista, los miembros de la comunidad mixtepequense de Abasolo del Valle, Veracruz, enviaron una delegación de jinetes de caballos de paso fino para participar como entretenimiento público durante la fiesta. Y, en 2016, los mayordomos acordaron no sacrificar todos los gallos del ritual de la despescuezada de gallos, para mejor regalarlos a la gente de la comunidad como respuesta a una solicitud en consideración a los derechos de los animales.

Los procesos de transformación en la fiesta de San Juan Bautista también están presentes en su práctica en la comunidad de diáspora en Bakersfield, California. Aproximadamente en el año 2000, los mixtepequenses de Bakersfield recibieron una réplica de la imagen de San Juan Bautista de la iglesia de San Juan Mixtepec, como un regalo por parte del ayuntamiento. La imagen fue transportada desde Oaxaca hacía la frontera en Tijuana donde fue "cruzada al otro lado" por una delegación de representantes de San Juan Mixtepec y de Bakersfield. Desde del momento en que esta imagen llegó a la iglesia local en Arvin, California, los migrantes mixtepequenses comenzaron a celebrar su propia fiesta de San Juan Bautista.

Figura 3

Disfrazados y público bailando durante la fiesta de San Juan Bautista en Bakersfield, CA

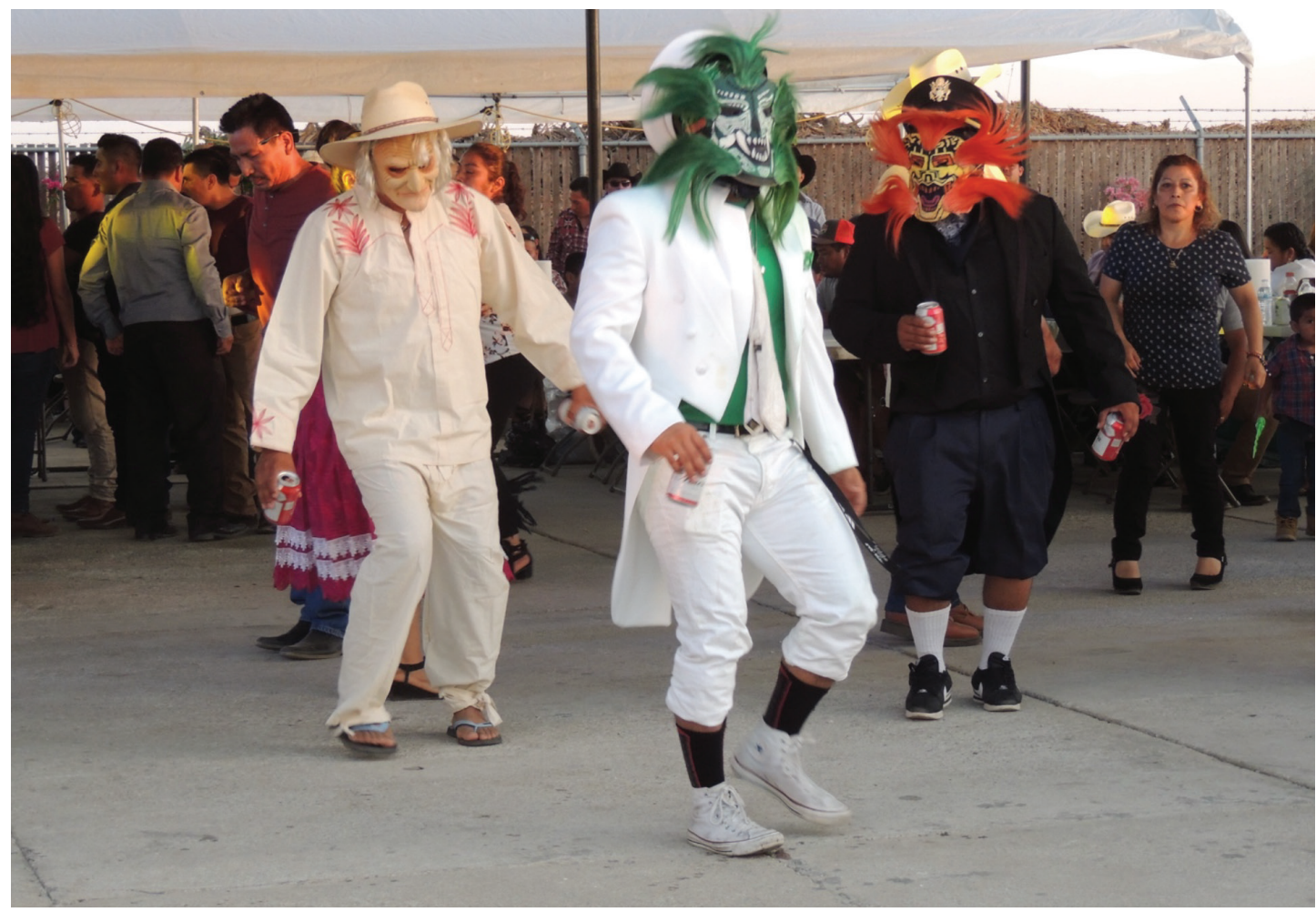

Fuente: Fotografía tomada por la persona autora en Bakersfield, CA, junio del 2017.

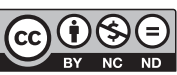


Aunque la fiesta se lleva a cabo aproximadamente durante las mismas fechas que la fiesta original, su organización y sus actividades difieren. La fiesta en diáspora no es un asunto de tres días sino de una sola tarde, los participantes disfrutan una comida tradicional de barbacoa y masa y un baile con disfrazados (Figura 3). En principio, cada detalle de esta fiesta es un reflejo de la original, pero obviamente muestra diferencias motivadas por los cambios del contexto geográfico y social, resultado de la migración transnacional. No está presente el ritual de la "despescuezada de gallos", la fiesta no dura varios días por cuestiones laborales y no hay una mayordomía al frente de la organización. Sin embargo, los migrantes mixtepequenses siguen practicando esta tradición como una parte de su identidad cultural, aunque estén lejos de su lugar de origen.

Figura 4

Los disfrazados tradicionales de San Juan Mixtepec

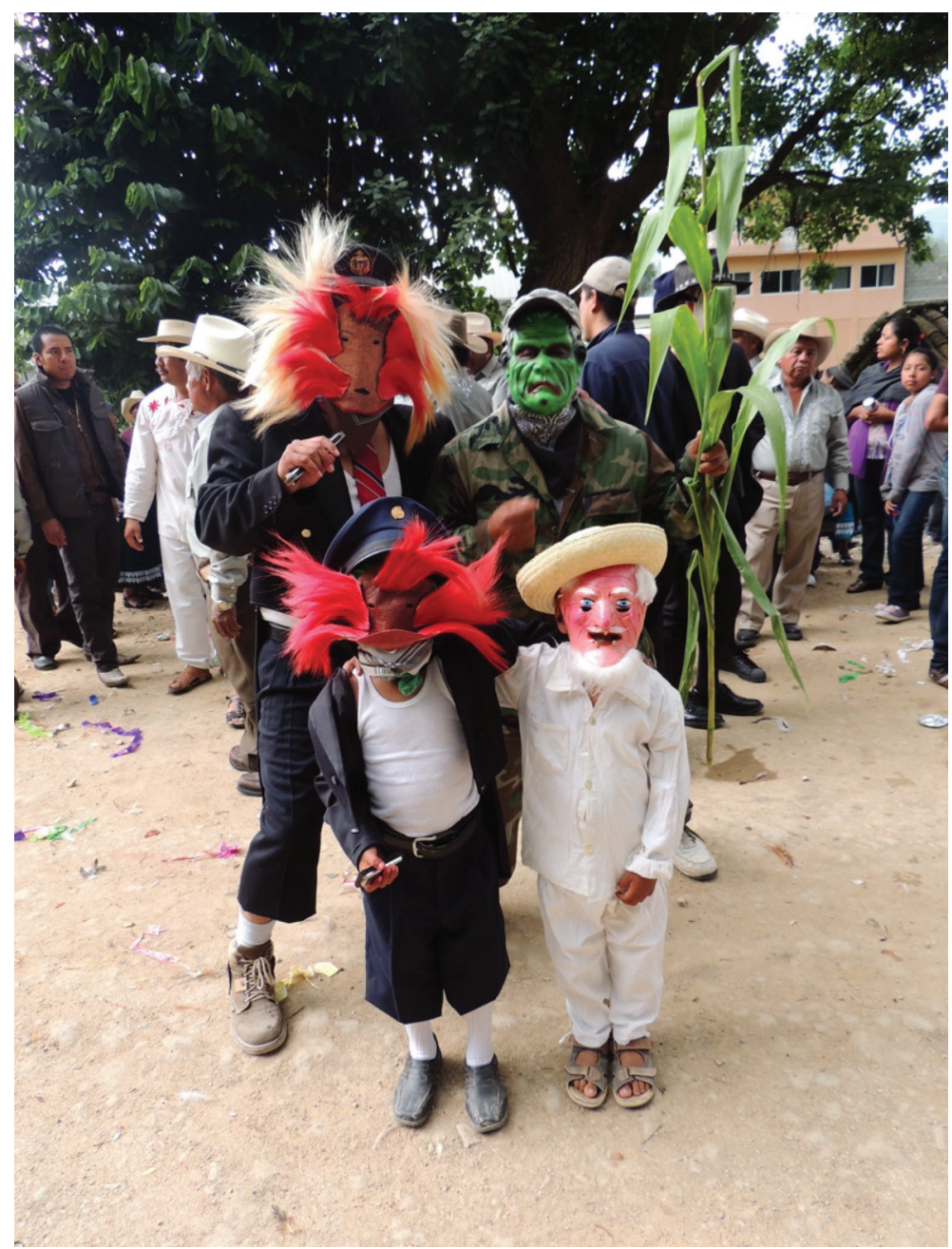

Fuente: Fotografía tomada por la persona autora en San Juan Mixtepec, junio de 2013.

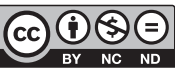


Otro ejemplo que muestra la naturaleza camaleónica de la práctica de tradiciones en San Juan Mixtepec son los disfrazados (Figura 4). Ahora bien, los cuatro tipos de disfrazados "tradicionales" son 1) el ñana chaa (el catrín); 2) el ñana ñaa (un personaje con atributos típicos de una mujer mixtepequense en términos de vestimenta, jugado exclusivamente por parte de hombres); 3) el tatsanu (el anciano); y, 4) el natsanu (la anciana). Cada personaje cuenta con una serie de características específicas de comportamiento, vestimenta, pasos de baile, música de harmónica y lenguaje usado en mixteco. Típicamente, los participantes aprenden sobre esta tradición a través de una imitación de lo que observan hacer a sus familiares o amistades. Además, cualquier miembro de la comunidad, sea hombre, mujer, niño o migrante, puede participar como disfrazado.

Pero, algunos interlocutores han observado cambios en las características del disfraz -lo que algunos atribuyen a la migración - siendo lo más extremo la aparición de diferentes personajes relativos a la celebración de Halloween, el desconocimiento de las canciones tradicionales de harmónica y el abandono del uso y de la producción de máscaras de madera en favor de máscaras de plástico.

Sin embargo, aunque algunos talvez no conocen los orígenes históricos ni los significados simbólicos asociados con cada uno, su participación cuenta como un acto de preservación cultural y una expresión de sentimientos acerca del orgullo de ser mixtepequense. Entonces, se puede deducir que la representación de los disfrazados durante fiestas específicas representa un transmisor corporal de discursos culturales acerca de lo que cuenta, y lo que no cuenta, como tradición para los mixtepequenses y los miembros de la audiencia. Esto es evidente en la práctica de los disfrazados fuera del ciclo tradicional de fiestas, como en situaciones de diáspora o, más recientemente, en grupos de preservación cultural quienes hacen performances de los disfrazados en distintos contextos sociales en otras partes de Oaxaca.

En años recientes ha surgido una iniciativa entre varios miembros de la comunidad para establecer grupos formales de preservación cultural, enfocados particularmente en la música tradicional, las fiestas y los disfrazados. Desde 2016, por ejemplo, el grupo cultural Ñana Cha'a Nana - Ña'a se ha dedicado a la investigación y la difusión de la tradición de los disfrazados en varias comunidades y ciudades dentro y fuera de Oaxaca. Sus miembros son residentes permanentes y migrantes repatriados, quienes están interesados en la preservación de los disfrazados. Antes de la creación de su grupo, está tradición era practicada exclusivamente durante ciertas fiestas específicas, pero ahora puede ser vista por parte de un público amplio en eventos culturales, en cualquier temporada del año. Además, este grupo -y también los mayordomos- han establecido concursos, durante ciertas fiestas como Carnaval y la fiesta de San Pedro, para premiar al "mejor" disfraz, en un intento por combatir la generalización de los personajes y promover un "rescate" de las prácticas tradicionales de la comunidad.

Estas convocatorias comenzaron a raíz de que algunas personas observaron muchas anomalías entre los participantes de las fiestas cuyo disfraz no seguía las características tradicionales de los personajes típicos, pues usaban otro tipo de prendas, máscaras de plástico en lugar de máscaras de madera, desconocían los pasos de danza o las melodías de harmónica, o inventaban personajes genéricos que recuerdan más a los disfraces de Halloween.

El hecho de mezclar o improvisar, no necesariamente nació desde la pereza, sino que varios de los participantes desconocían las características de los personajes típicos, algo que se atribuyó a una brecha generacional en la comunicación oral, o a la distancia física producto de la migración. No obstante, hay que notar aquí que, el hecho de presentar a los disfrazados fuera de su contexto original también representa una desviación de la tradición "auténtica", donde la apariencia de estos personajes se encuentra asociada exclusivamente con las fiestas que coincidían con una celebración del inicio del ciclo agrícola y la llegada de la temporada de lluvias, dos fechas importantes en el calendario cosmológico mixteco.

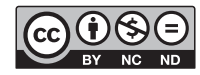


Sin embargo, los esfuerzos del grupo Ñana Cha'a Ñana - Ña'a indudablemente apoyan la importancia del concepto de preservación para la supervivencia de las tradiciones en el presente.

En el caso de la fiesta patronal y los esfuerzos del grupo cultural Ñana Cha'a Nana - Na'a se puede afirmar que plantean la naturaleza dinámica de las tradiciones y, particularmente, de factores sociales como la migración que influyen en su práctica actual. De hecho, se puede inferir que la práctica continua de la tradición depende, en muchos sentidos, no solamente de las interpretaciones variables de los participantes, sino de los contextos donde se llevan a cabo dichos eventos.

Esta diversidad de perspectivas y espacios permite una continuidad de las prácticas asociadas con las tradiciones festivas en el presente, pero adaptada al contexto particular social en que actualmente viven los mixtepequenses. Por supuesto, esto manifiesta el concepto de cambio, el cual abre la posibilidad de transformaciones en las tradiciones. Sin embargo, esto no debería ser considerado un atributo negativo, porque, como se verá en las siguientes conclusiones, la tradición de las fiestas, en todas sus manifestaciones, es un elemento importante en la formación de identidades y de los sentidos de pertenencia para los residentes y migrantes de San Juan Mixtepec.

\section{Conclusiones}

La práctica de tradiciones en las comunidades indígenas contemporáneas, tales como las prácticas festivas de los mixtepequenses presentadas en este artículo, registran la importancia de la permanencia y de la transformación como factores importantes para el concepto de tradición. Como se mencionó anteriormente, las maneras en que muchos mixtepequenses practican sus tradiciones festivas dependen de la transmisión oral y de la participación activa. La mayoría de la información histórica sobre las fiestas es compartida a través de estos dos medios y forma parte intrínseca en la continuidad de estas tradiciones tanto en Oaxaca como en la diáspora.

Sin embargo, una de las principales conclusiones de este artículo es que la tradición es un concepto camaleónico, lo que significa, en efecto, que parte de la naturaleza de las tradiciones es, precisamente, su disposición a la transformación. De esta manera, se puede decir que las tradiciones, tal como se practican en este contexto indígena particular, cuentan con un atributo "histórico", principalmente en el sentido de que existe un repertorio, basado en la memoria colectiva, de prácticas específicas asociadas con ciertas fiestas en particular. Las tradiciones están transmitidos a través de la repetición periódica de dichas prácticas en un contexto público, el contexto festivo, donde los participantes pueden observar, y luego replicar, estas prácticas corporizadas. No obstante, todas estas prácticas también están sujetas a una interpretación individual y contextual, la cual implica una característica dinámica.

Es así que, propongo que la tradición reside en el espacio entre estos dos conceptos de transformación y de permanencia -si se puede decir que es, realmente, un concepto que los participantes mismos manejen- y es un elemento esencial en la manera en que los mixtepequenses construyen sus sentidos de pertenencia. En efecto, la práctica continua de las tradiciones festivas, así como la nostalgia asociada con los recuerdos de las fiestas anteriores, impulsa su mantenimiento y su transformación. Los sentidos de pertenencia son construidos y mantenidos de diferentes maneras: 1) a través de la participación en, o la observación de, la mayordomía, 2) a través de los disfrazados, 3) a través de las prácticas performativas como los rituales y procesiones y, por supuesto, 4) a través de la presencia física en el contexto festivo, donde uno puede hablar en mixteco, probar los platillos tradicionales y bailar las danzas típicas, entre otras actividades.

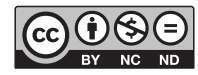


Esta mezcla de prácticas, olores, colores, música, sensaciones y experiencias forman una parte importante de cómo los mixtepequenses construyen quiénes son basados en un concepto de tradición, el cual vive y se mantiene en el presente. Sentirse "parte de" la comunidad mixtepequense implica, entonces, una interacción directa o indirecta con sus tradiciones; desde la participación en los cargos tradicionales hasta el consumo de videos y fotografías de las fiestas cargados en Internet. La construcción de los sentidos de pertenencia asociada con una identidad mixtepequense también forma parte de la experiencia migratoria, en el sentido de que los migrantes migran las tradiciones y las fiestas hacia sus comunidades de diáspora, así como, su regreso a Oaxaca forma parte clave de la continuación del ciclo festivo.

Lo que significa "ser" mixtepequense sigue siendo marcado por la práctica de las tradiciones, en este caso, específicamente, las tradiciones festivas. Además, estos sentidos de pertenencia también manifiestan la naturaleza multifacética del concepto de pertenencia, lo cual es vivido de varias maneras, dependiendo de las experiencias individuales de vida de los miembros de la comunidad. Desde la antropología se debe siempre considerar esta naturaleza dinámica de la tradición y no obsesionarse con una búsqueda de la "autenticidad". Las tradiciones siempre cambian, así como las formas en las que las personas pueden identificar lo que cuenta y lo que no cuenta como parte de las características de sus identidades y de sus sentidos de pertenencia.

Sin embargo, se puede identificar que el gran logro de la "tradición" en este contexto etnográfico específico es que este concepto sirve como catalizador para la construcción de sentidos de pertenencia en una comunidad indígena migratoria, aunque las experiencias cotidianas de las personas estén marcadas, en muchos sentidos, por el movimiento. Es así que, la tradición vive actualmente entre la permanencia y la transformación, dando una base histórica o conmemorativa a las experiencias y a los recuerdos de la gente, y al mismo tiempo sirve como un contexto dinámico para una exploración pública de novedosas construcciones de identidades.

\section{Notas}

1. Tomé dos niveles del mixteco en el Instituto Welte de Estudios Oaxaqueños en Oaxaca durante 2009 y 2011. El programa estaba patrocinado por parte del Centro de Estudios Latinoamericanos de San Diego State University, California y becado a través del Foreign Language and Area Studies Program (FLAS) de la United States Department of Education.

2. "Los mixtepequeses" o "los mixtepecanos" son términos auto-asignados y activamente usados por parte de los mismos miembros de la comunidad.

3. Secretaría de Desarrollo Social (SEDESOL). (2013). Datos generales del municipio San Juan Mixtepec - Dto. 08. Recuperado de http://www.microrregiones.gob.mx/zap/datGenerales. aspx?entra=zap\&ent=20\&mun=208.

4. El "tequio" se refiere a un sistema voluntario basado en servicio comunitario y en las estructuras sociales mixtepequenses de reciprocidad donde las personas residentes y migrantes de la comunidad participan por lo largo de sus vidas en diferentes proyectos, obras, comités, instituciones, etcétera asociadas con el bienestar de la comunidad y sus miembros.

5. La mayordomía representa el lado religioso de la jerarquía (también conocido como el "cargo") civil-religiosa en San Juan Mixtepec. El término "mayordomía" se refiere a la institución en

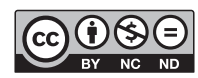


sí, así como los grupos particulares donde participan las personas encargadas, conocidas como "mayordomos", de santos y vírgenes específicos. Cada grupo de voluntarios forma una mayordomía, como la mayordomía de Cristo o la mayordomía de San Juan Bautista. Sus miembros sirven en el cargo por un año, y las obligaciones formales del mismo empiezan en la fecha de la entrega del cargo, la cual normalmente se lleva a cabo entre tres o cinco meses antes del inicio de las actividades festivas y termina entre tres o cinco meses después del fin de la fiesta. Además, muchos mayordomos se mantienen cerca de la institución después de su entrega para dar consejos o ayudar a los nuevos mayordomos quienes han asumido el cargo. Los participantes también trabajan en conjunto con las autoridades municipales, quienes representan el lado civil de la jerarquía civil-religiosa, durante ciertas fiestas.

6. Los comités forman parte del tequio comunitario. Todos los Mixtepequenses mayores de edad están obligados socialmente a participar en algún momento de sus vidas en uno o varios de los comités como el: del agua, del drenaje, de la educación, de la luz, etcétera; y ocupan este cargo voluntario por un año completo.

7. Las fiestas religiosas que celebran en San Juan Mixtepec son: la Virgen de Dolores, la Virgen de Guadalupe, la Virgen del Carmen, la Virgen de la Soledad, Cristo, el Santo Entierro, San José, San Sebastián, San Miguel Arcángel, San Pedro, Padre Jesús, San Salvador y el santo patrón San Juan Bautista.

8. Las fiestas seculares que celebran en San Juan Mixtepec son: Kastalenche (Carnaval), Día de la Independencia y Día de los Muertos.

9. En el original: "If tradition is a people's creation out of their own past, its character is not static but continuity; its opposite is not change but oppression, the intrusion of a power that thwarts the course of development".

10. En el original: "tradition refers both to the process of handing down from generation to generation, and some thing, custom, or thought process that is passed on over time".

11. El cargo se refiere a un peso, deber u obligación social y económica regulado a través de un sistema jerárquico de servicio comunitario lo cual implica una inversión fuerte en términos de tiempo, dinero y energía, muchas veces involucrando al participante por lo menos por un año en el servicio a su comunidad. Los cargos pueden ser religiosos (las mayordomías) o civiles (los comités).

12. En el original: "the quality of being traditional is a perception among participants that their action is the result of social connections that have endured through space and across time".

13. Término mixteco que significa "hombre de la palabra sagrada". Se refiere a ciertos hombres mayores de la comunidad quienes hablan palabras y discursos sagrados en mixteco antes de cualquier evento público, incluyéndose a las fiestas. Ellos comúnmente ofrecen bendiciones antes de bodas, bautismos, ceremonias cívicas, proyectos del tequio, fiestas religiosas y rituales.

14. En el original: "a process that evokes the traditional past not merely as part of a general dialogue with the past, but as part of an attempt to provide authority for one's own narrative performance and interpretation by supporting or contributing to a community's sense of what is 'traditional"'.

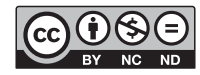


15. En el original: "refers to an interpretative process that embodies both continuity and discontinuity".

16. Este ritual resalta en particular la importancia de hacer un sacrificio colectivo para el beneficio simbólico de la comunidad entera. Cada mayordomo y cada miembro del cabildo municipal cuelgan de las patas un gallo adornado en papel maché, el cual queda suspendido entre dos postes de aproximadamente tres metros de altura. Jinetes montados a caballo pasan y arrancan violentamente las cabezas de los gallos y las lanzan a la multitud. Los participantes en este ritual lo describen como algo que honra al santo patrón de la comunidad, San Juan Bautista, y como una práctica sincrética que honra a los dioses de la lluvia en la cosmovisión prehispánica mixteca (Rieger, 2015).

\section{Referencias}

Anttonen, Pertti J. (2016). Tradition through Modernity. Postmodernism and the Nation-State in Folklore Scholarship. Helsinki: Finnish Literature Society.

Arévalo, Javier Marcos. (2004). La tradición, el patrimonio y la identidad. Revista de estudios extremeños, volumen 60 (3), 925-956.

Ben-Amos, Dan. (1984). The Seven Strands of Tradition: Varieties in its Meaning in American Folklore Studies. Journal of Folklore Research, volumen 21 (2/3), 97 - 131.

Besserer, Federico y Michael Kearney. (Eds.). (2006). San Juan Mixtepec. Una comunidad transnacional ante el poder clasificador y filtrador de las fronteras. México: Universidad Autónoma Metropolitana.

Cornelius, Wayne A., David S. FitzGerald, Jorge Hernández-Díaz y Scott Borger (Eds.). (2011). Migracíon desde la Mixteca: Una comunidad transnacional en Oaxaca y California. México: Miguel Ángel Porrúa.

Cornelius, Wayne A., Micah Gell-Redman, Hillary S. Kosnac, Pedro Lewin Fischer y Verónica Noriega (Eds.). (2017). El nuevo rostro de la migración mexicana: Una comunidad transnacional en Yucatán y California. México: Miguel Ángel Porrúa.

Edinger, Steven T. (1985). Camino de Mixtepec: Historia de un pueblo en las montañas de la Mixteca y su encuentro con la economía norteamericana. Fresno: Asociación Cívica Benito Juárez.

Glassie, Henry. (1995). Tradition. The Journal of American Folklore, volumen 108 (430), 395 - 412.

Graburn, Nelson H.H. (2001). What is Tradition? Museum Anthropology, volumen 24 (2/3), 6-11.

Handler, Richard y Jocelyn Linnekin. (1984). Tradition, Genuine or Spurious. The Journal of American Folklore, volumen 97 (385), 273-290.

Hernández-Díaz, Jorge. (2011). La migración en Oaxaca, una mirada a vuelo de pájaro. En Wayne Cornelius, David S. FitzGerald, Jorge Hernández-Díaz y Scott Borger (Eds.), Migracíon desde la Mixteca: Una comunidad transnacional en Oaxaca y California. México: Miguel Angel Porrúa.

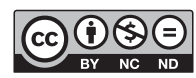


Herrejón Peredo, Carlos. (1994). Tradición. Esbozo de algunos conceptos. Relaciones, volumen 15, (59), $135-149$.

Hobsbawm, Eric y Terence Ranger. (Eds.). (1983). The Invention of Tradition. Cambridge: Cambridge University Press.

Horner, Alice E. (1990). The Assumption of Tradition: Creating, Collecting, and Conserving Cultural Artifacts in the Cameroon Grassfields (West Africa). (Tesis doctoral inédita). University of California, Berkeley.

Howard, Robert Glenn. (2013). Introduction: Vernacular Authority in Everyday Conversation. En Trevor J. Blank y Robert Glenn Howard (Eds.), Tradition in the Twenty-First Century: Locating the Role of the Past in the Present. Boulder: University of Colorado Press.

Joyce, Arthur A. (2010). Mixtecs, Zapotecs, and Chatinos: Ancient Peoples of Southern Mexico. Oxford: Wiley-Blackwell.

Lestage, Françoise. (2011). Los mixtecos en Tijuana. Reterritorialización y construcción de una identidad colectiva. México: El Colegio de la Frontera Norte.

Madrazo Miranda, María. (2005). Algunas consideraciones en torno al significado de la tradición. Contribuciones desde Coatepec (9), 115 - 132.

Mould, Tom. (2005). The Paradox of Traditionalization: Negotiating the Past in Choctaw Prophetic Discourse. Journal of Folklore Research, volumen 42, (3), 255 - 294.

Nagengast, Carole y Michael Kearney. (1990). Mixtec Ethnicity: Social Identity, Political Consciousness, and Political Activism. Latin American Research Review, volumen 25, (2), 61-91.

Noyes, Dorothy. (2009). Tradition: Three Traditions. Journal of Folklore Research, volumen 46, (3), $233-268$.

Ó Giollán, Diarmuid. (2000). Locating Irish Folklore: Tradition, Modernity, Identity. Cork: Cork University Press.

Ravicz, Robert S. (1965). Organización social de los mixtecos. México: Instituto Nacional Indigenista.

Rieger, Ivy A. (2015). Where the Clouds Descend: Fiestas and the Practice of Belonging in San Juan Mixtepec, Oaxaca, Mexico (Tesis doctoral inedita). University of Colorado, Boulder.

Rieger, Ivy A. (2017). La metamorfosis de la serpiente en la cultura mixteca. En Claudia Carranza Vera, Arturo Gutiérrez del Ángel y Héctor Medina Miranda (Eds.), La figura de la serpiente en la tradición oral iberoamericana. España: Fundación Joaquín Díaz.

Rieger, Ivy A. (2018). Memoria, pertenencia y la práctica de las fiestas en una comunidad mixteca. Boletín de Antropología, volumen 33, (56), 184-204.

Ruvalcaba, Daniela Oliver y Cristian Torres Robles. (2012). Excluidos y ciudadanos. Dimensiones del poder en una comunidad transnacional mixteca. México: Casa Juan Pablos.

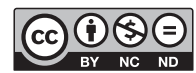


Terraciano, Kevin. (2001). The Mixtecs of Colonial Oaxaca: Ñudzahui History, Sixteenth through Eighteenth Centuries. Stanford: Stanford University Press.

Toren, Christina. (1988). Making the Present, Revealing the Past: The Mutability and Continuity of Tradition as Process. Man, volumen 23, (4), 696 - 717.

Velasco Ortíz, Laura. (2005). Mixtec Transnational Identity. Tuscon: The University of Arizona Press. 
